\title{
UN CONFRONTO RICORRENTE NELLA CULTURA CATTOLICA: IL CASO GALILEI ED IL CASO ROSMINI
}

\author{
Luciano Malusa
}

\begin{abstract}
RIASSUNTO: Le espressioni "caso Galilei" e "questione galileiana" inducono gli studiosi a riflettere su un'analoga condanna, emessa dagli organismi repressivi della Chiesa cattolica, circa duecentocinquant'anni dopo la condanna di Galilei, contro un sacerdote il quale aveva cercato una conciliazione tra l'avanzamento nella riflessione filosofico-scientifica e la via della tradizione. Antonio Rosmini-Serbati fu condannato nel 1887 con il Decreto Post obitum del Santo Uffizio. A tale sentenza egli non poté sottomettersi come Galilei, in quanto era morto da ormai trentadue anni. Rosmini, prima della sua dipartita (1855), aveva avuto dalla Chiesa il dispiacere di una condanna all'Indice di due sue opere (1849); aveva tuttavia ricevuto l'assoluzione su tutta la linea circa la sua produzione filosofico-teologica (1854). Dopo la morte i suoi avversari impugnarono questa decisione ufficiale della Chiesa, riuscendo a farlo condannare. Anche per Rosmini gli storici parlano di "questione rosminiana", e di "caso Rosmini". Nel presente articolo si confrontano le dimensioni delle due "questioni", e si rileva che la "riabilitazione" di Rosmini è passata attraverso una Nota della Congregazione per la Dottrina della Fede (2001), mentre nessun pronunciamento ufficiale ha "riabilitato" Galilei, verso il quale la cultura cattolica è stata largamente favorevole.
\end{abstract}

PAROLE CHIAVE: Galilei. Rosmini. Questione rosminiana. Questione galileiana. Santo Uffizio, Chiesa cattolica.

\section{I “CASI” di Galileo Galilei e di ANTonio Rosmini a Confronto sUl finire DELL'OTTOCENTO}

Nei giorni 26-30 maggio 2009 si è celebrato, con una complessa organizzazione, a Firenze, un Convegno internazionale dedicato a Il "caso Galileo". Un rilettura storica, filosofica, teologica. La partecipazione degli studiosi e del pubblico a questa iniziativa partita dal fiorentino (e gesuitico) "Centro Stensen” è stata notevole, a sottolineare l'importanza sempre maggiore che viene assegnata alla posizione di Galileo Galilei, considerata insieme come quella di costruttore di metodologie e come quella di un mediatore tra

\footnotetext{
${ }^{1}$ Professore ordinario di Storia del Cristianesimo e delle Chiese. Università degli Studi di Genova. Dipartimento di Antichità, Filosofia, Storia, Geografia (DAFIST). luciano.malusa@unige.it
} 
l'atteggiamento degli scienziati e la comunità cristiana dei fedeli. Non solo Galilei giganteggia nella storia del pensiero scientifico, ma è ormai valutato come un rilevante esponente della cultura cristiana. La sorte che gli è toccata, quella di dovere, proprio lui, credente e convinto della conciliabilità della ricerca scientifica in avanzamento e la tradizione religiosa, esegetica, etica e ascetica in consolidamento, sopportare la durezza di una condanna, è stata commentata dal mondo degli storici e degli scienziati con diversi accenti. Si è studiata la questione galileiana negli sviluppi che ha avuto nei secoli successivi e nel presente ${ }^{2}$.

Questo approfondimento, avvenuto per ricordare l'anniversario delle scoperte astronomiche di Galilei, relativamente ai satelliti di Giove (1609), non costituisce un episodio isolato. Da tempo la revisione operata dalla Chiesa cattolica e il riconoscimento di un errore compiuto dalle Congregazioni romane con la condanna del 1633 è servita a catalizzare gli studi sul "caso Galilei”. Si pensi che a Torino, in occasione dei vent'anni dalla morte di Luigi Firpo, si è svolto, con eccellenti risultati, un convegno dal titolo: Il processo a Galileo Galilei e la questione galileiana. Convegno, di impronta marcatamente laica, cui sono intervenute quasi tutte le stesse persone che hanno tenuto relazioni nel convegno fiorentino ${ }^{3}$. Fatto veramente significativo questa sorta di concorrenza tra iniziative sulla stesso argomento: il caso Galilei, cioè le motivazioni imperscrutabili ancora oggi su una così grave condanna.

L'uso delle espressioni "caso Galilei" e "questione galileiana" ha fatto riflettere il sottoscritto su un'analoga condanna, comminata dalla Chiesa nei suoi organismi repressivi circa duecentocinquant'anni dopo la condanna di Galilei, contro un esponente della cultura cattolica che egualmente aveva ritenuto fondamentale cercare una conciliazione tra l'avanzamento nella riflessione filosofico-scientifica e la via maestra della tradizione cattolica: intendo riferirmi alla sorte toccata ad Antonio Rosmini-Serbati, condannato nel 1887 con il Decreto Post obitum del Santo Uffizio, cui non poté sottomettersi al modo di Galilei, in quanto da ormai

${ }^{2}$ Il Convegno si è svolto in Firenze, in tre giornate, preceduto dall'inaugurazione in Santa Croce, il 26 maggio (alla presenza del Capo dello Stato); e concluso da una tavola rotonda in Arcetri, il 30 maggio. Di particolare rilievo per l'approfondimento del caso di Galilei nell'Ottocento sono state le relazioni di Bucciantini, Beretta, Ciliberto, Melloni. Cf. BUCCIANTINI, M.; CAMEROTA, M.; GIUDICE, F. (Eds.). Il caso Galileo, una rilettura, storica, filosofica, teologica. Firenze: Olschki, 2011.

${ }^{3}$ Il convegno torinese si è svolto il 26-27 marzo 2009, quindi con l'anticipo di due mesi rispetto alla manifestazione fiorentina. Vi sono stati interventi di numerosi studiosi, tra i quali ricordiamo: Paolo Galluzzi, Adriano Prosperi, William Shea, Francesco Beretta, Massimo Bucciantini, Germana Ernst, Annibale Zambarbieri. Cf. BRAVO, M.; FERRONE, V. (Eds.). Il processo a Galileo Galilei e la questione galileiana. Roma: Edizioni di Storia e Letteratura, 2010. 
trentadue anni egli era morto ${ }^{4}$. La condanna di Rosmini avveniva, curiosamente ma non troppo, in un contesto temporale in cui già si iniziava a pensare che la condanna dell'opera galileiana sui massini sistemi dell'Universo fosse stata un errore. Rosmini, in vita, aveva avuto dall'Istituzione ecclesiastica il dispiacere di una condanna: la Congregazione dell'Indice aveva proibito due sue opere, entrambe pubblicate nel 1848, Delle cinque piaghe della Santa Chiesa e La costituzione secondo la giustizia sociale, con una discutibile sentenza ottenuta da procedure che fanno venire i brividi 5 . L'anno prima di morire Rosmini aveva ricevuto dalla stessa Congregazione dell'Indice un'assoluzione su tutta la linea circa la sua produzione filosofico-teologica, sentenza che certo lo aveva consolato, ma che fu impugnata dai suoi avversari dopo la morte 6 . Anche per Rosmini gli storici parlano di "questione rosminiana”, per l'ovvio motivo che ad un'assoluzione piena circa la sua produzione filosofico-teologica (1854) seguì una forte condanna, diversi anni dopo la morte del pensatore (1887), che riguardava in parte proposizioni contenute in opere apparse postume, ma in parte opere già esaminate dalla Congregazione dell'Indice e non colpite da censure.

Quale tarda postilla, con cinque anni in ritardo, al Convegno galileiano di Firenze, nel corso del quale mi ero occupato, con una relazione, delle

${ }^{4}$ Gli atti del processo presso il Santo Uffizio, culminato con la condanna del 14 dicembre 1887 , solo più tardi resa nota con una lettera a tutti i vescovi $(7$ marzo 1888), sono stati pubblicati per le mie cure e di due collaboratori, traendoli dall'Archivio della Congregazione per la Dottrina della Fede: Antonio Rosmini e la Congregazione del Santo Uffzio. Atti e documenti inediti della condanna del 1887, ed. crit. a c. di MALUSA, L.; DE LUCIA, P.; GUGLIELMI, E. Milano: FrancoAngeli, 2008.

${ }^{5}$ Condanna comminata il 30 maggio 1849 dalla Congregazione dell'Indice, riunita in via straordinaria a Napoli, i cui atti sono stati integralmente pubblicati in: Antonio Rosmini e la Congregazione dell'Indice. Il decreto del 30 maggio 1849, la sua genesi ed i suoi echi, ed. crit. a c. di MALUSA, L. Stresa: Edizioni Rosminiane, 1999.

${ }^{6}$ Questa "assoluzione" degli scritti rosminiani avveniva alla fine di un procedimento, che viene chiamato dagli storici "esame delle opere", in quanto si trattò di un accurato esame di essi da parte di ben otto consultori della Congregazione dell'Indice. L'esame ebbe inizio con un decreto papale che imponeva il "silenzio" tra gli studiosi ed i religiosi che si erano affrontati circa le accuse a Rosmini di eterodossia dottrinale (1851). Un esame tanto ampio ed accurato non era cosa comune nella storia della Congregazione dell'Indice, inflessibile custode dell'ortodossia nelle pubblicazioni a stampa. Esso si concluse con un decreto da parte della Congregazione (comunemente detto Dimittantur) nel quale si "dimettevano" le opere esaminate non essendosi in esse trovato elemento di eterodossia. Sulla completa ricostruzione di questo "processo" il sottoscritto si sta muovendo, cercando di riportare alla luce integralmente gli atti dell'esame degli scritti rosminiani. L'intenzione sarebbe quella di pubblicare gli scritti di coloro i quali ritennero di non rinvenire nulla di pericoloso per la fede nella vastissima produzione del pensatore e lo scritto di quel consultore che, diversamente dagli altri, propose di condannare il pensiero del filosofo. Per conoscere le linee essenziali di questo esame delle opere cf. [PAGANI JR., G. B.]. Vita di Antonio Rosmini scritta da un Sacerdote dell'Istituto della Carità. Torino: Unione Tipografico-Editrice, 1897. Cito dall'edizione riveduta ed aggiornata da G. Rossi, Manfrini, Rovereto 1959, v. 2, p. 363-417. 
interpretazioni degli esponenti del neotomismo sul "caso Galilei" , intendo accennare alle polemiche scatenatesi sul finire dell'Ottocento intorno alla condanna del pensiero di Antonio Rosmini. Ho ripreso testi e schemi preparati per la relazione di allora, ma tralasciati per questioni di spazio, in quanto l'occasione di scrivere un saggio in onore dell'amico Gregorio Paia era troppo ghiotta per lasciarmi sfuggire di mano un intervento su una questione disputata, riguardante il destino di due grandi pensatori, accomunati da gravi difficoltà con la Chiesa cattolica. L'intreccio potrà, spero, piacere al festeggiato, entro la cornice di studi storico-filosofici raffinati, quali lui preferisce ${ }^{8}$.

Il parallelo che fu istituito tra il "caso Galilei" ed il "caso Rosmini" scaturiva da una serie di considerazioni ovvie, ma si sosteneva anche per una sorta di opposizione da parte di quei neotomisti di fine Ottocento che avevano in un certo senso "assolto" Galilei a fare altrettanto per Rosmini". Come mai, parecchi si chiesero, si tende ammette che la condanna dello scienziato toscano potrebbe essere "tolta" dalla fama di lui, con adeguato riconoscimento, quindi, della sua ortodossia cattolica, ed invece la condanna del filosofo roveretano, frutto di passioni post-risorgimentali, infondata sul terreno metafisico e teologico, viene mantenuta, con grave danno, tra l'altro, per l'Istituto della Carità da lui fondato?

La chiave di volta della questione era l'atteggiamento del movimento neotomista intransigente. I neotomisti condussero nel corso della loro battaglia per l'egemonia nella cultura cattolica, tra i pontificati di Pio IX e Leone XIII,

${ }^{7}$ Cf. MALUSA, L. Il giudizio dei neotomisti dell'Ottocento su Galileo Galilei. In: Il caso Galileo, p. 403-23. Questa relazione era stata "anticipata" in un articolo: Il dibattito ottocentesco sull'astronomo pisano. Galileo, Tommaso e i gesuiti, L'Osservatore romano, 27 maggio 2009, p. 4.

${ }^{8} \mathrm{Mi}$ si permetta con questa nota di dire quanto mi sia caro il professor Piaia, della cui amicizia mi onoro da circa quarantacinque anni. Lui si è affaticato per tutta la sua carriera negli studi storicofilosofici che hanno avuto il medioevo, il Rinascimento, l'età moderna, l'Illuminismo e l'Ottocento come periodi di riferimento importanti, senza dimenticare lo stesso Novecento e questi nostri ultimi anni. Si tratta di una vastità di interessi veramente notevole. Sulla storia della storiografia filosofica ha dato contributi rilevanti, a partire dagli studi fondamentali sulla storiografia filosofica di lingua francese tra Seicento ed Ottocento, per "discendere" nei secoli medievali. Pertanto l'amico Gregorio dovrebbe gradire questa specie di saggio che si configura come contributo alla "storia degli effetti" relativa a pensatori come Galilei e Rosmini. Non si tratta di un'indagine su aspetti marginali della presenza di Galilei nell'Ottocento italiano, ma di un raffronto sugli argomenti a favore o contro l'"ortodossia" di pensatori cristiani controversi ma preziosi per la Chiesa come Galilei e Rosmini.

${ }^{9}$ Per un chiarimento su questo parallelo cf. il lavoro del rosminiano BERTAMINI, Tullio. Galilei e Rosmini. In OTTONELlO, P.P. (Ed.): Rosmini e l'enciclopedia delle scienze, Atti del Congresso internazionale. Firenze: Olschki, 1998, p. 241-67. Cf. anche GALATI, D. Galileo e Rosmini. Roma: Kairos Edimedia, 1996 (il volume è dedicato solo in parte ad un raffronto). 
anche una lotta senza risparmio di colpi contro i pensatori rosminiani, al fine di convincere dell'insussistenza e della pericolosità insieme del sistema delineato da Antonio Rosmini, il quale continuava ad essere professato e discusso anche parecchi anni dopo la morte del filosofo. Mentre il pensatore di Rovereto viveva gli attacchi violenti all'ortodossia del suo sistema erano stati condotti per lo più da esponenti della Compagnia di Gesù, appoggiati dalla Curia romana e da un "partito" di cardinali e vescovi ostili o diffidenti nei confronti delle idee cattolico-liberali. Dopo la morte di Rosmini la diffusione notevole della sua originale prospettiva metafisica, chiarita anche dalla pubblicazione postuma della Teosofia, oltre che il permanere dell'influsso delle sue idee politiche, indussero l'ingresso in campo dei neotomisti. Colui il quale di distinse maggiormente sotto il profilo anche "mediatico" fu Giovanni Maria Cornoldi $^{10}$. Questa lotta fu poi voluta ed appoggiata da papa Leone XIII ${ }^{11}$ : si arrivò in tal modo alla condanna solenne da parte dalla Congregazione del Santo Uffizio, con il Decreto Post obitum, di quaranta proposizioni estratte da parecchie opere rosminiane, particolarmente da quelle postume ${ }^{12}$.

Questo provvedimento fu contestato da diversi esponenti del cattolicesimo, soprattutto in Italia, i quali paragonarono il "caso Rosmini" al "caso Galilei", considerando paradigmatiche di un modo di concepire l'ortodossia nei cattolici le due condanne clamorose contro pensatori i quali erano rei solamente di aver proposto posizioni speculative e scientifiche forse in anticipo sui propri tempi.

Delle proteste dei rosminiani e di diversi pensatori cattolici vicini allo spiritualismo si occupò il Santo Uffizio, il quale raccolse un voluminoso dossier

${ }^{10}$ Sul pensiero di questo dinamico intransigente gesuita cf. MALUSA, L. Neotomismo e intransigentismo cattolico, vol. I: Il contributo di Giovanni Maria Cornoldi per la rinascita del tomismo. Milano: IPL, 1986; vol. II: Testi e documenti per un bilancio del neotomismo. Gli scritti inediti di Giovanni Maria Cornoldi (Autobiografia - La controversia con Angelo Secchi), ivi 1989.

${ }^{11} \mathrm{Su}$ tale diretto intervento papale cf. MALUSA, L. Leone XIII, il Santo Uffizio e la questione rosminiana, in: La filosofia cristiana tra Ottocento e Novecento e il magistero di Leone XIII (Atti del Convegno - Perugia, 29 maggio - $1^{\circ}$ giugno 2003), Perugia: Curia Arcivescovile, 2004, p. $225-$ 55. Cf. anche, sempre di MALUSA, l'Introduzione a: Antonio Rosmini e la Congregazione del Santo Uffizio, p. 30-41.

${ }^{12}$ Il decreto, ricordiamo, porta la data del 14 dicembre 1887, ma venne pubblicato ed inviato ai vescovi in data 7 marzo 1888 (non a caso allora festa liturgica di San Tommaso). Cf. la versione del decreto pubblicata in CC, s. XIII, vol. X, 1888, p. 63-78. Il testo del decreto e le proposizioni rosminiane si trovano ora pubblicate, nelle diverse stesure redatte dal Santo Uffizio, in: Antonio Rosmini e la Congregazione del Santo Uffizio, p. 239-314. Sulla vicenda complessiva dei processi a Rosmini, cf. Dizionario dell'Inquisizione, diretto da PROSPERI, A., con la collaborazione di LAVENIA, V. TEDESCHI, J. Pisa: Edizioni della Normale, 2010, v. 3, p. 1341-7. 
degli interventi, in vista di prendere qualche provvedimento. In seguito però non si ritenne di dover agire, essendosi placate le proteste ${ }^{13}$. Richiamandomi soprattutto alla documentazione che ho consultato presso l'Archivio della Congregazione per la Dottrina della Fede, ho cercato qualche risposta agli atteggiamenti dissimili e strani dell'Istituzione e dei neotomisti, allora punta intransigente "ufficiosa" della Chiesa cattolica.

\section{IL RAFFrONTO TRA LE MODALITÀ E LE RAGIONI DELLE DUE CONDANNE}

Mi sembra interessante esaminare il parallelo Galilei-Rosmini, per cercare di capire le ragioni che i neotomisti contrapposero ai rosminiani onde negare ogni analogia tra i due casi. Il punto di polemica più aspro riguardò innanzitutto l'infondatezza filosofica e scientifica. Notevoli analogie si pensò si potessero riscontrare tra le due condanne e la personalità dei due pensatori. Soprattutto alcuni autori seguaci del rosminianesimo ritennero che la condanna fosse stata provocata da un atteggiamento di pregiudizio contro Rosmini, che prescindeva dall'erroneità delle sue proposizioni, non dimostrata e non dimostrabile, e che aveva indotto i giudici a calcare la mano su frasi ed espressioni isolate dal loro contesto, caricate di un significato erroneo che non avevano. Moltissime furono le pubblicazioni in cui si contestò la validità della solenne condanna ${ }^{14}$.

Debbo dire subito a scanso di equivoci che questa interpretazione dei filo-rosminiani ha trovato conferma con la pubblicazione degli atti dei processi contro Rosmini e mediante un approfondimento storiografico delle polemiche, in altre parole da uno studio accurato della "questione rosminiana"15. Potrei far notare come gli studi attualmente notevoli sulle condanne di Galilei non siano ancora arrivati ad alcune certezze sul comportamento dei giudici dello scienziato, mentre invece gli studi, certo meno numerosi, sulla questione rosminiana, e sui tre processi subiti da Rosmini, sono pervenuti ad alcuni punti

${ }^{13} \mathrm{Cf}$. il faldone del Santo Uffizio dedicato a queste proteste: ACDF, St.St. L 1-c bis.

${ }^{14}$ Per un elenco completo degli interventi intorno al decreto cf. BERGAMASCHI, C. Bibliografia rosminiana (1821-1915). Milano: Marzorati, 1974, p. 266-317. Cf. anche l'elenco delle polemiche sorte in MALUSA, L. Neotomismo e intransigentismo cattolico, I, p. 392-3.

${ }^{15}$ Cf. la già citata pubblicazione degli atti del primo processo intentato contro Rosmini, che portò alla condanna dell'opera Delle cinque piaghe della Santa Chiesa: Antonio Rosmini e la Congregazione dell'Indice. Il decreto del 30 maggio 1849, la sua genesi ed i suoi echi, ed. crit. a c. di MALUSA, L. Stresa: Edizioni Rosminiane, 1999. Cf. poi la pubblicazione degli atti del processo davanti al Santo Uffizio: Antonio Rosmini e la Congregazione del Santo Uffizio, cit. (in particolare sul lavoro della Congregazione le p. 41-52). 
fermi. In più, dal punto di vista ufficiale della Chiesa-Istituzione, è arrivata una preziosa precisazione, una Nota della Congregazione per la Dottrina della Fede, la quale, avendo ereditato dal Santo Uffizio la questione rosminiana e la condanna del 1887, puntualizza ufficialmente che la condanna va letta come un avviso agli studiosi onde non interpretare le quaranta proposizioni in un senso panteistico, ontologistico ed immanentistico ${ }^{16}$.

Partendo da questo documento ufficiale e guardando indietro nelle vicende di fine Ottocento, possiamo intendere anche meglio la proposta di riabilitazione del pensiero di Galilei, che scaturisce dalla constatazione che periodicamente le Istituzioni inquisitive romane hanno compiuto atti di repressione e di condanna che rispecchiano solo un momento storico particolare. In fondo si potrebbe essere d'accordo con l'interpretazione data da padre Angelo Secchi al momento storico della Chiesa in cui l'adesione al sistema copernicano stava diventando massiccia, ma in cui sembrava difficile conciliarlo con la fede cristiana e soprattutto accettarlo con tranquillità come la visione del mondo vera e dimostrata. La spiegazione della posizione centrale della Terra e del moto degli astri attorno ad essa aveva appassionato e turbato l'umanità. L'ipotesi di Copernico aveva permesso di pensare in modo diverso dall'apparenza senza rinnegare tuttavia l'osservazione e di diritti di un corretto ragionare. Vediamo cosa scrive Secchi: «Riusciti vani i tentativi per movimenti concentrici, gl'ingegni si rivolsero alle orbite eccentriche, ma a tutti i tentativi di questa specie faceva grande difficoltà il trovar modo da tenere la Terra fuori dal centro del mondo. Le menti non trovarono pace su questo punto, se non quando Copernico fece comprendere che l'esser la Terra rotonda non portava per necessaria conseguenza che essa fosse il centro del tutto, ma come semplice centro di una massa attraente [...] Stabilito così non esser mestieri che la Terra fosse fissa nel centro del mondo, prese consistenza il concetto emesso già in confuso da gran tempo di una Terra girante attorno ad un altro centro, che a sua volta si tenne fisso, cioè il Sole. Ma di questa conseguenza l'umano intelletto restò spaventato. Benché fosse poco conosciuta la vera distanza della Terra dal Sole, e appena si supponesse uguale ad un centesimo del vero, pure la dimensione del suo grand'orbe era sterminata, e tuttavia dalle ricerche fatte da

${ }^{16}$ Cf. Nota sul valore dei Decreti dottrinali concernenti il pensiero e le opere del Rev.do Sacerdote Antonio Rosmini Serbati. L'Osservatore romano, $1^{\circ}$ luglio 2001 (firmata dal Prefetto Joseph Ratzinger e dal Segretario Tarcisio Bertone). La Nota è stata pubblicata anche in un numero speciale di Charitas, luglio 2001, p. 206-10. Ricordo che il $1^{\circ}$ luglio 2001 ricorreva il centoquarantaseiesimo anniversario della morte del Roveretano. Si veda quale commento alla nota: MALUSA, L. "La Nota" della Congregazione per la Dottrina della Fede e gli studi rosminiani. Rivista Rosminiana, v. 96, p. 371-97, 2002. 
Galileo sopra le stelle risultava che questa immensa linea svaniva, e diventava un semplice punto veduto dalle stelle fisse [...] Lo spirito umano, in una parola, si spaventò quasi dell'opera sua, e per qualche tempo, e finché le prove non furono ineluttabili, cercò sfuggire da queste conseguenze trincerandosi dietro sacre parole, interpretando in modo, che di poi si riconobbe fallace» ${ }^{17}$. La condanna del copernicanesimo significò un momento di smarrimento; del resto Galilei non offriva soluzioni decisive, e quindi appariva ai più pericoloso.

La condanna delle quaranta proposizioni era arrivata pure essa in un momento di crisi per il pensiero cristiano, di fronte agli attacchi delle dottrine immanentistiche e materialistiche e nel contesto di una lotta degli Stati liberali contro la Chiesa, accusata di non accettare le libertà moderne. In questo contesto la condanna di Rosmini diventava un mezzo per fermare le adesioni di parecchi intellettuali cattolici ad una visione che poteva apparire incerta sia sotto il profilo sia metafisico che teologico e politico. Consapevoli di questa posizione assunta dalla Chiesa ufficiale, e soprattutto convinti che fosse stato consumato un atto di prepotenza e di inganno, i rosminiani premevano perché si arrivasse ad una pronta revisione della condanna, che tanto assomigliava a quella inflitta allo scienziato toscano ${ }^{18}$. Due momenti di disorientamento nella Chiesa cattolica, il primo agli inizi del Seicento, il secondo sul finire dell'Ottocento, avevano propiziato due errori delle Istituzioni inquisitive della Chiesa. Occorreva che ora i cristiani si rendessero conto della necessità di riconoscere questi errori.

Per capire la mentalità dei pensatori rosminiani e il loro atteggiamento di critica nei confronti degli esponenti della Chiesa-Istituzione citerei le pagine di Giuseppe Morando, scritte nella Introduzione alla sua vasta analisi delle quaranta proposizioni: Esame critico delle XL proposizioni rosminiane ${ }^{19}$. Mi debbo limitare

${ }^{17}$ SECCHI, A. Lezioni elementari di fisica terrestre, coll'aggiunta di due discorsi sopra la grandezza del creato. Torino-Roma: Loescher, 1879, p. 189-90.

${ }^{18}$ Cf. DE NARDI, P. Galileo Galilei e Antonio Rosmini davanti alla Romana Inquisizione. Foligno: Campitelli, 1889; ALESSIO, F. Haec meditare!, Il Bollettino rosminiano, 3, p. 24-32, 1888 (periodico dei rosminiani di Rovereto); Parallelismo delle persecuzioni fatte subire al Galilei ed al Rosmini. Il Nuovo Rosmini, I, v. 1, p. 180-91, 205-17, 303-11, 380-8, 482-503, 623-4, 1889 (l'autore anonimo presenta analogie tra le accuse rivolte ai due pensatori riguardo alla loro posizione circa la teologia eucaristica). Questo articolo, uscito in più puntate, con forti accenti polemici, attribuisce le persecuzioni subite dai due all'azione negativa della Compagnia di Gesù.

19 MORANDO, G. Esame critico delle XL proposizioni rosminiane condannate dalla $S . R . U$. Inquisizione. Studi filosofico-teologici di un laico. Milano: Cogliati, 1905, "Introduzione”, p. LVILXXII. L'opera mastodontica (p. CXL-996) era già stata preparata negli anni Novanta del secolo XIX, ma non era stata pubblicata per prudenza. Si noti che l'espressione "studi filosofico-teologici di un laico" non era a caso: Morando infatti era stato condannato dall'Inquisizione per alcune prese di posizione nel 1889, quando ancora era novizio nell'Istituto della Carità. Aveva ritenuto di dover 
solo a questo autore, per molti altri ${ }^{20}$. Leggendo un'impegnativa affermazione della "Civiltà Cattolica» che suonava così: «Un esempio notissimo nella storia è il decreto della Congregazione del Santo Offizio (24 febbraio 1616) sulla dottrina copernicana propugnata da Galileo, decreto che fu poscia praticamente riformato. Ora ciò che è riformabile non è infallibile» ${ }^{21}$, Morando faceva notare che anche la condanna di Rosmini avrebbe potuto essere riformata. Così dicendo apriva una questione piuttosto delicata ${ }^{22}$.

Infatti il padre gesuita Eugenio Polidori che aveva fatto sulla «Civiltà Cattolica» l'affermazione impegnativa intendeva dire che l'infallibilità nella Chiesa spettava solo al papa quando parlava "ex cathedra» per sua iniziativa od in un Concilio. I decreti delle Congregazioni romane quindi non erano infallibili. Ma allora quale tipo di autorevolezza dovevano avere questi pronunciamenti? L'avallo del papa ai decreti della Congregazioni, con l'approvazione, assegnava ad essi grande autorevolezza, ma non dava il grado supremo di infallibilità. Dunque per Morando era chiaro che il caso Galilei doveva essere come di guida al fedele rosminiano nello sperare nella riforma della dura sentenza del Post obitum.

Per la verità Polidori aveva citato non tanto la condanna di Galilei, ma il decreto di condanna del copernicanesimo, provocato dall'azione del card. Bellarmino (1616). In questo caso quindi non si era condannata una persona (od un libro), ma una dottrina, dichiarando il copernicanesimo assurdo in filosofia ed eretico in teologia. Ora, per il gesuita, tale condanna non aveva più motivo di esistere, essendo del tutto assodata la verità della visione copernicana, anzi essendo stata poi tale visione ampliata e corretta da successive verifiche sperimentali. La condanna all'Indice del dialogo sui massimi sistemi dell'universo, e la condanna di Galilei per eresia, conseguente

uscire dalla congregazione dei Rosminiani per essere in grado, rimanendo laico, di lavorare a difesa dell'ortodossia di Rosmini, della quale era intimamente convinto.

${ }^{20}$ Cf. quali analisi delle quaranta proposizioni i seguenti contributi più rilevanti: Alle quaranta rosminiane proposizioni col Decreto "Post obitum" condannate. Milano: Cogliati, 1889 (inizialmente pubblicata nel Rosmini); BILLIA, L. M. Quaranta proposizioni attribuite ad Antonio Rosmini coi testi originali completi dell'Autore e con altri dello stesso che ne compiono il senso. Milano: Hoepli, 1889; F.C.D. [Anonimo] Ragioni della condanna fatta dal S. Uffizio delle cosi dette XL proposizioni di Antonio Rosmini. Firenze: Cellini, 1889. L'anonimato della maggior parte degli scritti si spiegava con il fatto che erano per lo più dei sacerdoti gli autori delle analisi critiche della condanna.

${ }^{21}$ POLIDORI, E. Per la critica storica. Un po' di teologia per tutti”. CC, s. XVIII, v. 5, 1902, p. 656. L'articolo occupa le p. 641-60: la prima puntata dell'articolo era stata in s. XVII, v. 5, 1899, p. 18 33 (dedicata però alle tematiche della certezza di posizioni della Chiesa come il culto delle reliquie).

${ }^{22}$ Cf. MORANDO, Introduzione, p. LVI-LVIII. 
a quel libro, erano egualmente riformabili, essendo state pronunciate proprio perché Galilei non aveva ottemperato all'intimazione di Bellarmino ad adeguarsi al decreto inquisitoriale. Morando faceva notare che, se questo era l'atteggiamento della "Civiltà Cattolica», non si capiva perché gli stessi Gesuiti si accanissero nel considerare la condanna delle quaranta proposizioni rosminiane come definitiva ${ }^{23}$.

La polemica ora ci interessa in quanto già alla fine del secolo XIX la Chiesa si dichiarava disposta a rivedere ed a riformare la condanna del copernicanesimo, non essendo infallibile il decreto inquisitoriale. Detta condanna invece non ha mai ricevuto una riforma e solo il solenne intervento di papa Giovanni Paolo II, nella sede del ricordo della sorte toccata a Galilei, ha precisato solennemente (ma senza i caratteri dell'infallibilità) che nel comportamento della Congregazione del Santo Uffizio (ed anche nelle proibizioni di quella dell'Indice) era insito il grave errore di considerare l'eliocentrismo come affermazione eretica ${ }^{24}$. Se nell'autorevole "Civiltà Cattolica» si scriveva di riformabilità delle sentenze delle Congregazioni, immaginarsi l'emozione dei rosminiani, che potevano sperare in un pronto intervento papale o al livello dei vescovi per richiedere al Santo Uffizio di rivedere il giudizio sulle quaranta proposizioni.

\section{Il DibATTITO SUlLA POSSIBILITÀ DI “RIFORMARE” I DECRETI DELL'AUTORITÀ INQUisitiva DELLA CHIESA}

Nella produzione di quegli anni di smarrimento e di rabbia da parte rosminiana fu invocato un precedente, nella storia della Chiesa, di cancellazione, avvenuta sul finire del Quattrocento, di una condanna per eresia, avendo riconosciuto il papa stesso Alessandro VI, che, nel condannare, le autorità inquisitive avevano agito con precipitazione. Era stato il caso della condanna delle novecento Conclusiones, proposizioni tratte da diverse correnti della sapienza e del pensiero antico e medievale, fatte stampare da Giovanni Pico della Mirandola in vista della disputa tra i dotti che il Signore

${ }^{23}$ Cf. quanto aveva asserito Cornoldi in alcuni suoi interventi: Soluzione della questione rosminiana. CC, s. XIII, v. 10, 1888, p. 257-78; Lorenzo Billia e il decreto "Post obitum", ivi, s. XIV, v. 4, 1889, p. $858-73$, v. 5,1890 , p. $47-58$.

${ }^{24}$ Cf. la notizia della "riabilitazione" compiuta da Giovanni Paolo II, con il discorso del 10 novembre 1979 davanti alla Pontificia Accademia delle Scienze, con un commento: La fede, la scienza e il "caso Galileo", CC, 1979, v. 4, p. 417-24. 
di Mirandola aveva indetto a Roma ${ }^{25}$. Dopo la severa condanna, nel 1487, il giovane nobile filosofo si era salvato con la fuga ed aveva trovato rifugio in Firenze grazie alla protezione di Lorenzo de' Medici. Il nuovo papa, Alessandro VI, aveva, a seguito di una trattativa con il Medici, "perdonato" il Signore di Mirandola e Concordia, annullando la sentenza di condanna. Tale atto di perdono e di annullamento della condanna venne ricordato, in un certo senso "ripescato" come esempio di un atto di revisione piuttosto rapido di una decisione dottrinale, che però non aveva le caratteristiche dell'infallibilità ${ }^{26}$.

La polemica su questo lontano episodio di "revoca" di una condanna dell'Inquisizione, si esaurì senza prese di posizione ufficiali. Nessun atto concreto di revisione venne dal pontificato di Pio X, nel corso del quale l'autorità papale si impegnò molto nella lotta antimodernistica. Una richiesta precisa fu fatta invece sotto il pontificato di papa Benedetto XV, da parte di un esponente dell'Istituto della Carità, Giambattista Pagani jr. ${ }^{27}$, il quale fece pervenire al papa una supplica per riformare il Post obitum, affermando che a venticinque e più anni di distanza dal decreto, si era potuto assodare che l'erroneità delle posizioni rosminiane era stata affermata a seguito di un confronto con la metafisica, la gnoseologia e la psicologia tomiste, e che quindi il decreto del Santo Uffizio in realtà aveva evidenziato delle difformità

${ }^{25}$ Cf. delle Conclusiones l'edizione a cura di BIONDI, A. Firenze: Olschki, 1995.

${ }^{26}$ Cf. su questo argomento i seguenti articoli: PAGANI, Giuseppe. Giovanni Pico della Mirandola condannato da Innocenzo VIII e prosciolto da Alessandro VI. Il Rosmini, III, 1889, v. 1, p. $232-$ 48; Prelato romano, Lettera di un Prelato romano aperta alla "Civiltà Cattolica", ivi, p. 670-80. La risposta che negava ogni riformabilità delle sentenze di condanna fu di CORNOLDI, G.M. Saggio della polemica di un prelato romano rosminiano, CC, s. XIV, v. 2, 1889, p. 257-82 e 285-404. La polemica proseguì negli anni con l'intervento della rivista Rassegna Nazionale, organo dei cattolici conciliatoristi. Cf. tra i diversi interventi: PAGANI, Giuseppe L'assoluzione di Giovanni Pico della Mirandola e la "Civiltà Cattolica". Pistoia: Ed. Rassegna Nazionale, 1899. Su tutti questi interventi: MALUSA, Neotomismo, I, p. 473-5.

27 Sulla vita e sulla personalità di Giovanni Battista Pagani Jr. (1844-1926), l'autore, tra l'altro, della monumentale Vita di Rosmini, qui in precedenza citata, scritta anonima "da un sacerdote dell'Istituto della Carità", nel 1896 (poi ripubblicata per Manfrini, Rovereto 1959), ci si deve limitare ad un necrologio contenuto nel Bollettino dell'Associazione Antonio Rosmini e dei Collegi rosminiani, 1926, n. 14, p. 23-4. La personalità di questo religioso meriterebbe molto di più, cioè uno studio sul suo impegno nel rivalutare costantemente la figura di Rosmini. Negli anni della scrittura del documento inviato a papa Benedetto XV, Pagani era Provinciale d'Italia dell'Istituto della Carità. Compì il suo ministero ed i suoi studi tra Borgomanero, dove era nato, e Domodossola, dove morì. Si cita il nome di questo studioso con l'indicazione di "junior" (jr.) per distinguerlo da un altro Giovanni Battista Pagani, pure lui di Borgomanero, uno dei primi Presbiteri dell'Istituto della Carità, che fondò l'attività dell'Istituto in Inghilterra e fu Preposito generale della Congregazione dopo la morte di Rosmini. 
delle dottrine di Rosmini da una certa interpretazione della dottrina di San Tommaso $^{28}$.

Quale idea poteva avere papa Benedetto delle decisioni prese dal Santo Uffizio, quasi trent'anni prima, sotto Leone XIII, a proposito di un filosofo di cui egli conosceva abbastanza poco? $\mathrm{Ci}$ si dovrebbe chiedere in che relazione potevano stare le affermazioni di papa Benedetto sulla moderazione nei dibattiti tra cristiani (in riferimento alla lotta antimodernista) con le vicende di una dibattito tanto sofferto quanto "gridato", che aveva visto un certo sfoggio di intransigenza da parte neotomista e di insofferenza da parte rosminiana ${ }^{29}$.

Il papa Benedetto XV, con un Motu proprio del 25 marzo 1917, intitolato De attribuenda Santi Officii causa librorum..., aveva ordinato la soppressione della Congregazione dell'Indice, ed il passaggio delle competenze di questa al Santo Uffizio ${ }^{30}$. Tale provvedimento mirava solo a "razionalizzare" la repressione entro la Chiesa, oppure era un atto di moderazione? Con questa ulteriore responsabilizzazione del Santo Uffizio si lasciava intravedere qualche revisione di sentenze? Nulla di tutto questo si verificò. La richiesta di revisione, inoltrata ufficialmente da parte di Pagani, non ebbe alcun seguito. Essa restò dimenticata negli incartamenti del Santo Uffizio dedicati alle reazioni nel campo cattolico al Post obitum ${ }^{31}$.

${ }^{28}$ L'istanza presentata da Pagani è stata da me rinvenuta in: ACDF, Fondo SO, L 1-c bis. Il documento, di 131 pagine in quarto, è anonimo, con la dicitura "Riservato", e porta quale titolo: Le quaranta Proposizioni del Decreto "Post Obitum" confrontate col testo di Antonio Rosmini e presentate alla Santità di Nostro Signore Benedetto XV. Cf. sul significato complessivo della condanna, in sintonia proprio con le considerazioni di Pagani, il mio saggio: L'ultima fase della questione rosminiana e il decreto "Post Obitum". Stresa: Sodalitas, 1989.

29 Sull'atteggiamento di Benedetto XV nei confronti delle lotte antimodernistiche, e poi del neotomismo e del rosminianesimo, cf. MALUSA, L. Lo studio della filosofia di San Tommaso nelle scuole cattoliche durante il pontificato di Benedetto XV, in MAURO, L. (ed.): Benedetto XV. Profeta di pace in un mondo in crisi. Bologna: Minerva, 2008, p. 239-270.

${ }^{30}$ Acta Apostolicae Sedis, IX, 1917, p. 167. Cf. ZANOTTI, A. Benedetto XV e il Codex Iuris Canonici, in: Benedetto XV, p. 167-79.

${ }^{31}$ Il rosminiano G.B. Pagani jr., prima di decidere l'intervento, che poi non ebbe esito, per riabilitare la memoria di Rosmini aveva dovuto attendere parecchi anni per divulgare l'edizione dell'Epistolario completo di Rosmini, in 13 voll., da lui curato (con altri) e pubblicato presso l'Editore Pane di Casale Monferrato tra il 1887 e il 1894. Sull'insieme di queste incredibili vicende che si possono configurare come una strisciante prosecuzione nei confronti dei Padri rosminiani si sofferma il volume: MALUSA, L.; ZANARDI, S. Le lettere di Antonio Rosmini-Serbati un "cantiere" per lo studioso. Introduzione all'epistolario rosminiano. Venezia: Marsilio, 2013, p. 15-25. Si segnala che è in corso di avanzata realizzazione la nuova edizione delle Lettere di Antonio Rosmini che completa e perfeziona l'edizione paganiana. Tale edizione critica diretta da P.P. Ottonello vedrà la pubblicazione di un volume primo dedicato alle lettere del periodo della formazione (1813-1816), per le cure di L. Malusa e S. Zanardi. 
La posizione ufficiale della Chiesa, in quegli anni in cui era stata combattuta l'eresia modernistica, quindi fu di considerare che le responsabilità dell'Istituzione inquisitoriale nella condanna del 1616 e del 1633 non dovevano essere troppo enfatizzate, in quanto spettava alle Congregazioni romane sorvegliare sull'ortodossia dottrinale e non era tanto semplice farlo. Occorreva fare salva la buona fede delle Congregazioni e la loro sollecitudine a proteggere dall'errore e dall'eresia i cristiani. Leggiamo sempre dalla "Civiltà Cattolica»: «Ma se le Congregazioni romane, nei loro giudizi, non sono infallibili; se, per ciò stesso, le loro sentenze dottrinali, anche in materie che riguardano la fede e i costumi, non sono irreformabili, segue forse che non vi sia alcun obbligo di accettare tali sentenze?» ${ }^{32}$. In un certo senso i gesuiti della "Civiltà Cattolica», per salvaguardare la rispettabilità della condanna delle proposizioni rosminiane apparivano restii agli inizi del Novecento ad accettare la revisione dei decreti anti-copernicani ed a riconoscere che nei confronti di Galilei era stato adoperata una vera persecuzione arbitraria.

I pensatori neotomisti in questi due casi si comportarono in modo contraddittorio, dichiarandosi disponibili alla revisione della condanna di Galilei, pur senza però insistere su di essa, e chiudendosi a riccio nel caso della più recente condanna di Rosmini, negando addirittura fondamento al dato storico della assoluzione di Pico della Mirandola ${ }^{33}$. Curiosamente la Chiesa, dovendo elevare Rosmini e non Galilei all'onore degli Altari ${ }^{34}$, ha preso, con la Nota del 2001 una posizione ufficiale per precisare la condanna del 1887 e invece ha lasciata indeterminata la riabilitazione dello scienziato toscano, non producendo alcun documento (attraverso la Congregazione per la Dottrina della Fede) che dicesse con chiarezza quello che prima abbiamo letto dalla penna di Secchi: in un momento di indecisione la Chiesa ha ritenuto di far precedere l'imperio alla distensione degli animi ed alla riflessione scientifica.

\section{Alcune Conclusioni}

Se si può tentare un minimo di conclusione circa l'atteggiamento del pensiero neotomistico dell'Ottocento (e di parte del Novecento) nei confronti

32 BRANDI, S. M. Per la verità. Un altro po' di teologia per tutti. CC, s. XVIII, v. 6, p. 389-390, 1902.

${ }^{33}$ Cf. di BRANDI: Un breve di Leone X citato dai rosminiani. CC, s. XVII, v. 5, p. 399-415, 1899; Pico della Mirandola e Antonio Rosmini. Risposta alla Rassegna Nazionale, ivi, v. 8, p. 322-32, 1899.

${ }^{34}$ Antonio Rosmini è stato beatificato con solenne cerimonia svoltasi in Novara, cioè nella Diocesi in cui egli è morto, il 18 novembre 2007. Cf. la presentazione dell' itinerario svoltosi per la sua proclamazione a Beato in: PAPA, C.M. Rosmini: conoscere e credere. Storia della causa. Roma: Studium, 2007. 
del pensiero di Galileo Galilei, e di riflesso nei confronti di quello di Rosmini, occorre dire che nessun tipo di campagna antigalileiana fu compiuto da parte dei pensatori che si rifacevano alla ripresa piena della filosofia dell'Aquinate. Il tentativo di conciliare la visione fisica ilemorfica con i principi della metafisica realistica e trascendente di Tommaso, principi non posti in dubbio dal pensiero moderno, od almeno da una parte di esso, si dimostrò fragile. Certamente la filosofia della materia e della forma aveva un suo fondamento ed alcuni elementi di profonda verità. Tuttavia il modo di applicare questa visione ai progressi della fisica dell'Ottocento si dimostrò inadeguato. Il fatto che alcuni esponenti di rilievo del pensiero cristiano, come ad esempio lo scienziato Secchi (ma, qualche decennio più tardi, lo scienziato e geologo Stoppani, guarda caso seguace dalla filosofia rosminiana), concepissero ampie riserve sulla visione ilemorfica, preferendo accettare il concetto di forze fisiche e di atomismo fisico-chimico, spiega di un disagio di fronte ad un'applicazione poco felice di certi concetti in origine metafisici, e originati da una visione qualitativa del cosmo ad una nuova realtà, quella determinata dallo sperimentalismo galileiano.

Il rispetto sostanziale della posizione galileiana da parte dei neotomisti non significò quindi la piena adesione alla sua concezione fisico-meccanica. Si sorvolò sul fatto che un pensiero cristiano poteva benissimo essere seguace della fisica nuova (e poi della nuova chimica) prescindendo, sul piano della scienza sperimentale, dai concetti, ampiamente vaghi, di materia, forma e forma sostanziale. Si sorvolò anche sul fatto che, proprio ai tempi di Galilei, diversi filosofi e teologi avevano ritenuto di compiere una conciliazione tra i dati fisici proposti dalle nuove osservazioni, e quindi una visione corpuscolare, e i dati metafisici scolastici ${ }^{35}$. La difesa della visione spiritualistica e realistica insieme della conoscenza poteva venire anche da chi accettava pienamente la visione sperimentalistica, e quindi si poteva essere in fondo più galileiani di quanto non lo fossero i neotomisti.

Sul versante delle problematiche poste dalla condanna di Galilei si nota nei fatti l'accettazione della tesi che lo scienziato toscano sbagliò a volere semplicemente sostenere la prospettiva eliocentrica sulla base di argomenti scritturali ed a volere inoltre forzare le posizioni del suo tempo nella direzione di una prova inconfutabile di essa. I neotomisti si astennero tuttavia accuratamente dal pronunciare parole di riprovazione della

${ }^{35}$ Cf. l'illustrazione di queste posizioni di "compromesso" nella "classica" opera di REDONDI, P. Galileo eretico. Torino: Einaudi, 1983 (più volte ripubblicata: l'ultima edizione presso Laterza, Roma-Bari 2009). 
posizione di Galilei: lo fece Secchi, difendendo i padri gesuiti del Seicento, su posizioni più avanzate a livello fisico-metafisico rispetto allo scienziato toscano. Tuttavia né Secchi né i neotomisti diminuirono il loro giudizio positivo sull'ortodossia di Galilei.

La quérelle sulla condanna di Galilei non toccò quindi che marginalmente i neotomisti, i quali furono in un certo senso provocati ad essa da una specie di "coda" delle polemiche rosminiane, in cui essi si erano distinti molto. Il parallelo tra le condanne di Galilei e quelle di Rosmini (adoperiamo appositamente il plurale) fu fatto da parte rosminiana dopo la pronuncia del Decreto Post obitum, in quanto apparve chiaro a molti pensatori cristiani che vi erano parecchie analogie nei metodi e nei contenuti di esse. Tuttavia il problema vero sollevato dai rosminiani non era di assimilare le posizioni di Rosmini a quelle di Galilei, bensì di invocare la fallibilità di decreti ufficiali e solenni come erano quelli del Santo Uffizio. Si pose la domanda cruciale del comportamento da parte della Chiesa di fronte ad un chiaro errore nel condannare un pensatore cristiano: se era divenuta evidente la macroscopicità dell'errore compiuto nel 1633, alla luce delle nuove risultanze delle scoperte astronomiche e fisiche, poteva apparire ad un esame spassionato del processo intentato a Rosmini e delle metodologie adoperate che l'errore c'era stato pure in questa sentenza.

Le analogie tra i due casi sono però più formali che sostanziali, e lo dimostra il fatto che non vi fu nel 2001, con la Nota della Congregazione per la Dottrina della Fede, alcuna ammissione di errore, relativamente al decreto Post obitum, ma una precisazione della portata della condanna, che venne confermata nei termini di una condanna delle tentazioni al panteismo ed all'ontologismo che potevano scaturire seguendo alcuni testi postumi del pensatore di Rovereto. Le affermazioni di papa Giovanni Paolo II, fatte all'inizio del suo pontificato, intorno al "caso Galilei", sembrano quasi "chiedere scusa" (anticipando quanto sarà fatto poi in vista dell'anno giubilare) alla comunità scientifica ed agli stessi cristiani per un atto di condanna che si dimostrò infondato sotto tutti i punti di vista, sia teologico, che filosofico e scientifico. Le precisazioni della Nota non presentano alcun atto di scusa, in quanto, formalmente, la "proscrizione" delle quaranta proposizioni viene presentata come una precauzione agli studiosi nell'interpretare in un certo modo il pensiero di Rosmini su quei temi che vengono presentati, estrapolandoli, da molteplici testi.

Nessun documento della Congregazione per la Dottrina della Fede ha avanzato precisazioni né sul decreto del 1616, né sulla condanna del 1633. Con 
il curioso risultato che la condanna delle quaranta proposizioni rosminiane non è stata per nulla riformata, ma solo precisata, mentre invece la condanna del 1633 è stata riconosciuta sbagliata, ma non è stata formalmente mai tolta. In entrambi i casi è apparsa con chiarezza la linea della Chiesa, di tenere conto che i propri passi talvolta possono essere anche incerti, evidenziando che il valore del messaggio rivelato e posto in essere da una plurisecolare tradizione deve essere sempre costantemente verificato e rinnovato. Verrebbe da commentare così: per quanto riguarda il decreto Post obitum meglio tardi che mai l'intervento della Congregazione nel 2001. Quando si erano avanzate delle perplessità da parte di molti e autorevoli pensatori cattolici (Morando e soprattutto G.B. Pagani) circa le modalità della condanna di Rosmini, visti i casi di Galilei e di Pico della Mirandola, si erse un muro di intransigenza, soprattutto neotomistica e gesuita, che non rappresenta certo una bella pagina per la storia della Chiesa.

MALUSA, Luciano. A recurrent comparison in Catholic culture: the case of Galilei and the case of Rosmini. Trans/Form/Ação, Marília, v. 37, p. 117-134, 2014. Ediçâo Especial.

\begin{abstract}
The expressions "the case of Galilei" and "the Galilean question" induce researchers to reflect on an analogous condemnation issued by the repressive bodies of the Catholic Church about two hundred and fifty years afterwards. This was the condemnation of a priest, Antonio RosminiSerbati, who had tried to reconcile tradition with advancement in philosophical-scientific reflection. Rosmini was condemned in 1887 by a Post obitum decree of the Holy Inquisition. He could not submit to that conviction as did Galilei, because by then he had been dead for thirty-two years. Before his death in 1855, Rosmini endured the disappointment of a condemnation in the Index of two of his works (1849), but later received absolution across the board for his philosophical and theological production (1854). After his death, his opponents contested this official decision and succeeded in having him convicted. Thus historians write about "Rosminian question", and "Rosmini case". In the present essay we compare the dimensions of the two "questions", and we note that the "rehabilitation" of Rosmini has gone through a Note of the Congregation for the Doctrine of the Faith in 2001, while no official pronouncement has "rehabilitated" Galilei.
\end{abstract}

KEYWORDS: Galilei. Rosmini. Rosminian question. Galilean question. Holy Inquisition. Catholic Church. 


\section{Bibliografia}

BERGAMASCHI, C. Bibliografia rosminiana (1821-1915). Milano: Marzorati, 1974.

BERTAMINI, Tullio. Galilei e Rosmini. In: OTTONELLO, P.P. (Ed.). Rosmini e l'enciclopedia delle scienze, Atti del Congresso internazionale. Firenze: Olschki, 1998, p. 241-267.

BRAVO, M.; FERRONE, V. (Eds.). Il processo a Galileo Galilei e la questione galileiana. Roma: Edizioni di Storia e Letteratura, 2010.

BUCCIANTINI, M.; CAMEROTA, M.; GIUDICE, F. (Eds.). Il caso Galileo, una rilettura, storica, filosofica, teologica. Firenze: Olschki, 2011. La fede, la scienza e il "caso Galileo". La Civiltà Cattolica (CC), v. 4, p. 417-424, 1979.

GALATI, D. Galileo e Rosmini. Roma: Kairos Edimedia, 1996.

MALUSA, L. Neotomismo e intransigentismo cattolico. Milano: IPL, 1986. 2v.

. "La Nota" della Congregazione per la Dottrina della Fede e gli studi rosminiani. Rivista Rosminiana, v. 96, p. 371-397, 2002.

. (A cura di) Antonio Rosmini e la Congregazione dell'Indice. Il decreto del 30 maggio 1849, la sua genesi ed i suoi echi. Stresa: Edizioni Rosminiane, 1999.

; DE LUCIA, P.; GUGLIELMI, E. (A cura di). Antonio Rosmini e la Congregazione del Santo Uffrzio. Atti e documenti inediti della condanna del 1887. Milano: FrancoAngeli, 2008.

; ZANARDI, S. Le lettere di Antonio Rosmini-Serbati: un "cantiere" per lo studioso. Introduzione all'epistolario rosminiano. Venezia: Marsilio, 2013

PAGANI JR., G. B. Vita di Antonio Rosmini: scritta da un Sacerdote dell'Istituto della Carità. Edizione riveduta ed aggiornata da G. Rossi. Rovereto: R. Manfrini, 1959. 2v.

REDONDI, P. Galileo eretico. Torino: Einaudi, 1983.

SECCHI, A. Lezioni elementari di fisica terrestre, collaggiunta di due discorsi sopra la grandezza del creato. Torino-Roma: Loescher, 1879. 
MALUSA, L. 